\title{
Front Matter: Volume 8938
}

, "Front Matter: Volume 8938," Proc. SPIE 8938, Optical Fibers and Sensors for Medical Diagnostics and Treatment Applications XIV, 893801 (7 March 2014); doi: 10.1117/12.2053714

SPIE. Event: SPIE BiOS, 2014, San Francisco, California, United States 


\section{PROGRESS IN BIOMEDICAL OPTICS AND IMAGING}

\section{Optical Fibers and Sensors for Medical Diagnostics and Treatment Applications XIV}

Israel Gannot

Editor

1-2 February 2014

San Francisco, California, United States

Sponsored and Published by

SPIE 
The papers included in this volume were part of the technical conference cited on the cover and title page. Papers were selected and subject to review by the editors and conference program committee. Some conference presentations may not be available for publication. The papers published in these proceedings reflect the work and thoughts of the authors and are published herein as submitted. The publisher is not responsible for the validity of the information or for any outcomes resulting from reliance thereon.

Please use the following format to cite material from this book:

Author(s), "Title of Paper," in Optical Fibers and Sensors for Medical Diagnostics and Treatment Applications XIV, edited by Israel Gannot, Proceedings of SPIE Vol. 8938 (SPIE, Bellingham, WA, 2014) Article CID Number.

ISSN: 1605-7422

ISBN: 9780819498519

Published by

SPIE

P.O. Box 10, Bellingham, Washington 98227-0010 USA

Telephone +1 3606763290 (Pacific Time) · Fax +1 3606471445

SPIE.org

Copyright (@ 2014, Society of Photo-Optical Instrumentation Engineers.

Copying of material in this book for internal or personal use, or for the internal or personal use of specific clients, beyond the fair use provisions granted by the U.S. Copyright Law is authorized by SPIE subject to payment of copying fees. The Transactional Reporting Service base fee for this volume is $\$ 18.00$ per article (or portion thereof), which should be paid directly to the Copyright Clearance Center (CCC), 222 Rosewood Drive, Danvers, MA 01923. Payment may also be made electronically through CCC Online at copyright.com. Other copying for republication, resale, advertising or promotion, or any form of systematic or multiple reproduction of any material in this book is prohibited except with permission in writing from the publisher. The CCC fee code is $1605-7422 / 14 / \$ 18.00$.

Printed in the United States of America.

Publication of record for individual papers is online in the SPIE Digital Library.

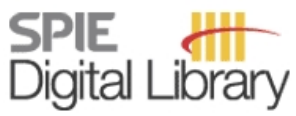

SPIEDigitalLibrary.org

Paper Numbering: Proceedings of SPIE follow an e-First publication model, with papers published first online and then in print and on CD-ROM. Papers are published as they are submitted and meet publication criteria. A unique, consistent, permanent citation identifier (CID) number is assigned to each article at the time of the first publication. Utilization of CIDs allows articles to be fully citable as soon as they are published online, and connects the same identifier to all online, print, and electronic versions of the publication. SPIE uses a six-digit CID article numbering system in which:

- The first four digits correspond to the SPIE volume number.

- The last two digits indicate publication order within the volume using a Base 36 numbering

system employing both numerals and letters. These two-number sets start with 00, 01, 02, 03, 04, $05,06,07,08,09,0 A, 0 B \ldots$. 0Z, followed by 10-1Z, 20-2Z, etc.

The CID Number appears on each page of the manuscript. The complete citation is used on the first page, and an abbreviated version on subsequent pages. Numbers in the index correspond to the last two digits of the six-digit CID Number. 


\section{Contents}

ix

Conference Committee

SESSION 1 OPTICAL FIBERS AND SENSORS I

$893804 \quad$ Needle-tip localization using an optical fibre hydrophone [8938-2]

J. M. Mari, Univ. College London (United Kingdom); S. West, Univ. College Hospital (United

Kingdom); P. C. Beard, A. E. Desjardins, Univ. College London (United Kingdom)

893805 Selenide and telluride glasses for mid-infrared bio-sensing (Invited Paper) [8938-3]

S. Cui, Univ. de Rennes 1 (France) and Zhejiang Univ. (China); R. Chahal, Y. Shpotyuk, C. Boussard, J. Lucas, Univ. de Rennes 1 (France); F. Charpentier, H. Tariel, DIAFIR (France); O. Loréal, V. Nazabal, Univ. de Rennes 1 (France); O. Sire, V. Monbet, Univ. Européenne de Bretagne (France); Z. Yang, The Univ. of Arizona (United States) and Australian National Univ. (Australia); P. Lucas, The Univ. of Arizona (United States); B. Bureau, Univ. de Rennes 1 (France)

893806 Effects of sterilization on optical and mechanical reliability of specialty optical fibers and terminations [8938-4]

A. A. Stolov, E. T. Warych, W. P. Smith, P. L. Fournier, A. S. Hokansson, J. Li, R. S. Allen, OFS

Specialty Photonics Div. (United States)

\section{SESSION 2 OPTICAL FIBERS AND SENSORS II}

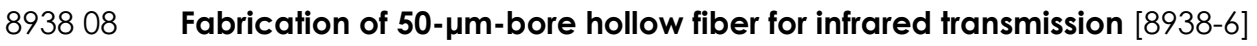

K. Iwai, K. Takahashi, H. Takaku, Sendai National College of Technology (Japan); M. Miyagi, Tohoku Institute of Technology (Japan); Y.-W. Shi, Fudan Univ. (China);

Y. Matsuura, Tohoku Univ. (Japan)

893809 Monitoring biofilm attachment on medical devices surfaces using hyperspectral imaging [8938-8]

H. N. D. Le, U.S. Food and Drug Administration (United States) and Johns Hopkins Univ. (United States); V. M. Hitchins, I. K. Ilev, D.-H. Kim, U.S. Food and Drug Administration (United States)

8938 OA Improved deep UV fiber for medical and spectroscopy applications [8938-9]

V. Khalilov, J. Shannon, R. Timmerman, Polymicro Technologies (United States)

8938 OB Design of a long working distance graded index fiber lens with a low NA for fiber optic probe in OCT application [8938-55]

X. Sun, J. Li, OFS, Specialty Photonics Div. (United States) 
8938 OE New glass developments for fiber optics [8938-12]

P. L. Higby, SCHOTT North America, Inc. (United States); K. Holst, SCHOTT AG (Germany);

K. Tabor, W. James, E. Chase, S. Pucilowski, E. Gober-Mangan, R. Klimek, SCHOTT North America, Inc. (United States); F. Karetta, SCHOTT AG Technical Services (Germany); B. Schreder, SCHOTT AG Advanced Optics (Germany)

8938 OG Improvement of optical damage in specialty fiber at $266 \mathrm{~nm}$ wavelength [8938-14] T. Tobisch, TransMIT GmbH (Germany); H. Ohlmeyer, Technische Hochschule Mittelhessen (Germany) and TransMIT GmbH (Germany); H. Zimmermann, S. Prein, CryLas GmbH (Germany); J. Kirchhof, S. Unger, IPHT Jena (Germany); M. Belz, TransMIT GmbH (Germany); K.-F. Klein, Technische Hochschule Mittelhessen (Germany) and TransMIT GmbH (Germany)

$8938 \mathrm{OH}$ Integrated optical fiber shape sensor modules based on twisted multicore fiber grating arrays [8938-15]

P. S. Westbrook, K. S. Feder, T. Kremp, T. F. Taunay, E. Monberg, OFS Labs. (United States);

J. Kelliher, OFS Specialty Fiber Div. (United States); R. Ortiz, OFS Labs. (United States);

K. Bradley, OFS Optical Connectivity Solutions (United States); K. S. Abedin, OFS Labs. (United States); D. Au, OFS Specialty Fiber Div. (United States); G. Puc, OFS Labs.

(United States)

\section{SESSION 4 OPTICAL FIBERS AND SENSORS IV}

8938 ol Silver-coated Teflon hollow waveguides for the delivery of terahertz radiation [8938-16] J. E. Melzer, Rutgers, The State Univ. of New Jersey (United States); M. Navarro-Cía, Univ. College London (United Kingdom) and Imperial College London (United Kingdom); O. Mitrofanov, Univ. College London (United Kingdom); J. A. Harrington, Rutgers, The State Univ. of New Jersey (United States)

8938 0J Multi-channel near-infrared spectrometer for functional depth-resolved tissue examination and positioning applications [8938-17]

D. Ernst, M. Peyer, D. Täschler, Bern Univ. of Applied Sciences (Switzerland); P. Steiner, Bern Univ. of Applied Sciences (Switzerland) and Univ. Bern (Switzerland); A. Bossen, B. Považay, Ch. Meier, Bern Univ. of Applied Sciences (Switzerland)

8938 OL Microstructured optical fiber-based micro-cavity sensor for chemical detection [8938-19] B. Kim, J.-C. Ahn, P.-S. Chung, Beckman Laser Institute Korea (Korea, Republic of) and Dankook Univ. (Korea, Republic of); Y. Chung, Gwangju Institute of Science and Technology (Korea, Republic of)

$8938 \mathrm{ON}$ Low-temperature, low-cost growth of robust ATR $\mathrm{GeO}_{2}$ hollow fibers based on copper capillary tubes for transmission of $\mathrm{CO}_{2}$ laser light [8938-21]

C. Jing, W. Bai, Z. Hu, P. Yang, East China Normal Univ. (China); A. Liu, F. Lin, Shanghai Normal Univ. (China); Y. Shi, Fudan Univ. (China); J. Chu, East China Normal Univ. (China)

\section{SESSION 5 OPTICAL FIBERS AND SENSORS V}

$89380 Q \quad$ Optical fiber spectroscopy measures perfusion of the brain in a murine Alzheimer's disease model [8938-24]

H. J. Ahn, S. Strickland, J. Krueger, D. Gareau, The Rockefeller Univ. (United States) 
8938 OR Quantifying temperature changes in tissue-mimicking fluid phantoms using optical coherence tomography and envelope statistics [8938-25]

S. Seevaratnam, A. Bains, M. Farid, Ryerson Univ. (Canada); G. Farhat, Univ. of Toronto (Canada) and Sunnybrook Health Sciences Ctr. (Canada); M. Kolios, B. A. Standish, Ryerson Univ. (Canada)

8938 OS Gold nanoparticles sensing with diffusion reflection measurement as a new medical diagnostics application (Invited Paper) [8938-7]

D. Fixler, Bar-llan Univ. (Israel)

8938 OT Bio-functionalized hollow core photonic crystal fibers for label-free DNA detection [8938-26]

A. Candiani, Univ. degli Studi di Parma (Italy); H. T. Salloom, Univ. of Baghdad (Iraq) and AlNahrain Univ. (Iraq); E. Coscelli, M. Sozzi, A. Manicardi, Univ. degli Studi di Parma (Italy);

A. K. Ahmad, Al-Nahrain Univ. (Iraq); A. H. Al-Janabi, Univ. of Baghdad (Iraq); R. Corradini,

G. Picchi, A. Cucinotta, S. Selleri, Univ. degli Studi di Parma (Italy)

\section{SESSION 6 OPTICAL FIBERS AND SENSORS VI}

8938 OU The efficacy of pressure relief maneuvers in spinal cord injury patients: a clinical study [8938-27]

T. Ho, A. T. Nguyen, The Catholic Univ. of America (United States); A. Lichy, S. Groah, National Rehabilitation Hospital (United States); J. C. Ramella-Roman, Florida International Univ. (United States)

8938 OV Towards mid-infrared fiber-lasers: rare earth ion doped, indium-containing, selenide bulk glasses and fiber [8938-28]

H. Sakr, Z. Tang, D. Furniss, L. Sojka, N. A. Moneim, E. Barney, S. Sujecki, T. M. Benson,

A. B. Seddon, The Univ. of Nottingham (United Kingdom)

8938 OW Towards mid-infrared supercontinuum generation: Ge-Sb-Se mid-infrared step-index small-core optical fiber [8938-29]

J. H. Butterworth, D. Jayasuriya, Q. Q. Li, D. Furniss, N. A. Moneim, E. Barney, S. Sujecki, T. M. Benson, The Univ. of Nottingham (United Kingdom); J. S. Sanghera, Naval Research

Lab. (United States); A. B. Seddon, The Univ. of Nottingham (United Kingdom)

8938 0X Exposed core microstructured optical fiber surface plasmon resonance biosensor [8938-30] E. Klantsataya, A. François, A. Zuber, The Univ. of Adelaide (Australia); V. Torok, The Univ. of Adelaide (Australia) and South Australian Research and Development Institute (Australia);

R. Kostecki, T. M. Monro, The Univ. of Adelaide (Australia)

\section{SESSION 7 OPTICAL FIBERS AND SENSORS VII}

8938 OY Hollow fiber based SERS probe for analysis of biological molecules [8938-31]

M. Nagaoka, T. Katagiri, Y. Matsuura, Tohoku Univ. (Japan)

893811 Spatial compound imaging for fiber-bundle optic microscopy [8938-34]

G. W. Cheon, J. Cha, J. U. Kang, Johns Hopkins Univ. (United States)

893813 Micro sized implantable ball lens-based fiber optic probe design [8938-36]

J. Cha, J. U. Kang, Johns Hopkins Univ. (United States) 
893814 CP-OCT sensor guided SMART micro-forceps [8938-37]

C. Song, Johns Hopkins Univ. (United States) and Daegu Gyeongbuk Institute of Science and Technology (Korea, Republic of); P. L. Gehlbach, Johns Hopkins School of Medicine (United States); J. U. Kang, Johns Hopkins Univ. (United States)

893815 Biconically tapered fiber optic dip probe for rapid label-free immunoassays [8938-38] J. Miller, A. Castaneda, K. H. Lee, M. Sanchez, S. Murinda, W.-J. Lin, E. Salik, California State Polytechnic Univ., Pomona (United States)

893816 Application of ball-lens hollow fiber Raman probe for studying anorectal prolapse [8938-39]

B. B. Andriana, A. Taketani, Kwansei Gakuin Univ. (Japan); C. L. R. Soeratman, The Univ. of Tokyo (Japan); M. Ishigaki, Y. Maeda, M. Sawa, H. Sato, Kwansei Gakuin Univ. (Japan)

893817 Chalcogenide microstructured optical fibers for chemical sensing [8938-40] J. Troles, P. Toupin, Glass and Ceramic Team, CNRS, Univ. de Rennes 1 (France); L. Brilland, PERFOS (France); C. Boussard-Plédel, B. Bureau, Glass and Ceramic Team, CNRS, Univ. de Rennes 1 (France); D. Mechin, PERFOS (France); J.-L. Adam, Glass and Ceramic Team, CNRS, Univ. de Rennes 1 (France)

893819 Label-free biochemical characterization of bovine sperm cells using Raman microscopy [8938-42]

A. C. De Luca, S. Managò, M. Ferrara, L. Sirleto, Consiglio Nazionale delle Ricerche (Italy); R. Puglisi, D. Balduzzi, A. Galli, Istituto Sperimentale Italiano Lazzaro Spallanzani (Italy); I. Rendina, P. Ferraro, G. Coppola, Consiglio Nazionale delle Ricerche (Italy)

8938 1A Large-core tube-leaky waveguide for delivery of high-powered Er:YAG laser [8938-43] S. Kobayashi, T. Katagiri, Y. Matsuura, Tohoku Univ. (Japan)

\section{POSTER SESSION}

8938 1B Full-field optical coherence tomography system implemented with fiber optic components [8938-44]

S. K. Lee, J. H. Kim, E. S. Choi, Chosun Univ. (Korea, Republic of)

$89381 \mathrm{E} \quad$ Cost-effective optical coherence tomography spectrometer based on a tilted fiber Bragg grating [8938-47]

S. Remund, A. Bossen, Bern Univ. of Applied Sciences (Switzerland); X. Chen, Bangor Univ. (United Kingdom); L. Wang, Jiangnan Univ. (China), Katholieke Univ. Leuven (Belgium), and NERF (Belgium); A. Adebayo, L. Zhang, Aston Univ. (United Kingdom); B. Považay, C. Meier, Bern Univ. of Applied Sciences (Switzerland)

8938 IF Blood pH optrode based on evanescent waves and refractive index change [8938-48] K. Hammarling, Mid Sweden Univ. (Sweden); J. Hilborn, Uppsala Univ. (Sweden); H.-E. Nilsson, A. Manuilskiy, Mid Sweden Univ. (Sweden)

$89381 \mathrm{~J} \quad$ Optical fiber-based photomechanical molecular delivery system [8938-52] K. Nakano, Tokyo Univ. of Agriculture and Technology (Japan); S. Sato, S. Kawauchi, H. Ashida, National Defense Medical College Research Institute (Japan); I. Nishidate, Tokyo Univ. of Agriculture and Technology (Japan) 


\section{1K Self-referenced label free biosensors based on differential fiber optic interferometry}

[8938-53]

R. B. Queirós, C. Gouveia, INESC TEC, Univ. do Porto (Portugal); M. C. L. Martins, Instituto de Engenharia Biomédica, Univ. do Porto (Portugal); P. A. S. Jorge, INESC TEC, Univ. do Porto (Portugal)

Author Index 
Proc. of SPIE Vol. 8938 893801-8

Downloaded From: https://www.spiedigitallibrary.org/conference-proceedings-of-spie on 26 Apr 2023 Terms of Use: https://www.spiedigitallibrary.org/terms-of-use 


\title{
Conference Committee
}

\author{
Symposium Chairs
}

James G. Fujimoto, Massachusetts Institute of Technology

(United States)

R. Rox Anderson, Wellman Center for Photomedicine, Massachusetts General Hospital (United States) and Harvard School of Medicine (United States)

Program Track Chairs

Tuan Vo-Dinh, Fitzpatrick Institute for Photonics, Duke University (United States)

Anita Mahadevan-Jansen, Vanderbilt University (United States)

\section{Conference Chair}

Israel Gannot, Tel Aviv University (Israel)

\section{Conference Program Committee}

James P. Clarkin, Polymicro Technologies, A Subsidiary of Molex Incorporated (United States)

Ilko llev, U.S. Food and Drug Administration (United States)

Jin U. Kang, Johns Hopkins University (United States)

Karl-Friedrich Klein, Technische Hochschule Mittelhessen (Germany)

Pierre Lucas, The University of Arizona (United States)

Yuji Matsuura, Tohoku University (Japan)

Angela B. Seddon, The University of Nottingham (United Kingdom)

Session Chairs

Keynote Address

Israel Gannot, Tel Aviv University (Israel)

1 Optical Fibers and Sensors I

Angela B. Seddon, The University of Nottingham (United Kingdom)

2 Optical Fibers and Sensors II

Pierre Lucas, The University of Arizona (United States)

3 Optical Fibers and Sensors III

Jin U. Kang, Johns Hopkins University (United States) 
4 Optical Fibers and Sensors IV

James A. Harrington, Rutgers, The State University of New Jersey

(United States)

5 Optical Fibers and Sensors $V$

Moinuddin Hassan, U.S. Food and Drug Administration (United States)

6 Optical Fibers and Sensors VI

Yuji Matsuura, Tohoku University (Japan)

7 Optical Fibers and Sensors VII

James P. Clarkin, Polymicro Technologies, A Subsidiary of Molex Incorporated (United States)

8 Optical Fibers and Sensors VIII

Karl-Friedrich Klein, Technische Hochschule Mittelhessen (Germany) 